\title{
Antiretroviral treatment and association with prematurity in perinatal HIV-exposed children
}

\author{
Ana Maria Tudor ${ }^{1,2^{*}}$, Mariana Mărdărescu2 ${ }^{2}$, loana Alina Anca ${ }^{1,3}, C^{2}$ istina Petre ${ }^{2}$, Ruxandra Neagu Drăgchicenoiu ${ }^{2}$, \\ Rodica Ungurianu², Dan Oțelea ${ }^{2}$, Simona Paraschiv² \\ From The 10th Edition of the Scientific Days of the National Institute for Infectious Diseases "Prof Dr Matei Bals" \\ Bucharest, Romania. 15-17 October 2014
}

\section{Background}

In the last years there is a great concern regarding the effect of HIV and antiretroviral drugs in children born by treated HIV-infected mothers.

We started a prospective cohort study regarding HIV and antiretroviral exposure in children followed in the Pediatric HIV Department from the National Institute for Infectious Diseases “Prof. Dr. Matei Balş”, Bucharest.

\section{Methods}

We analyzed the data recorded for children perinatallyexposed to HIV followed up in our Department from January $1^{\text {st }} 2006$ to December $31^{\text {st }} 2012$. The patients were followed up for 18 month after birth to establish the HIV status; gestational age, birth defects and mother treatment were noted.

\section{Results}

From 206 children with complete 18 months follow up, $21 \%$ (43 cases) were diagnosed with HIV infection and more than $33 \%$ had at least one congenital condition.

We found birth defects in various organs in studied children: heart (130 cases), musculoskeletal system (47 cases), kidney (20 cases), nervous system (20 cases), digestive tract (10 cases) and metabolic and genetic disorders (2 cases each). 26 from the 163 HIV-exposed children and 6 from the $43 \mathrm{HIV}$-infected cases were born before 37 weeks of gestation, 4 HIV-exposed and 4 $\mathrm{HIV}$-infected children were small for gestational age. We found low birth weight $(<2500 \mathrm{~g})$ in 18 HIV-exposed children and 3 HIV infected children and extremely low birth weight (1000) in one HIV-exposed child.
We found congenital malformation in 11 preterm HIV-exposed children and 2 preterm HIV-infected children, but also in $38 \mathrm{HIV}$-exposed children and 19 HIV-infected babies with normal gestation period.

The difference between the rate of congenital malformation and prematurity was not statistically significant $(\mathrm{p}=0.08)$ in any of studied groups and HIV diagnosis was not associated with a higher risk of preterm birth $(\mathrm{p}=0.93)$. Mother being part of the Romanian cohort was not statistically associated with higher risk of prematurity. We found a significant association between antiretroviral treatment during pregnancy and prematurity $(\mathrm{p}=0.003)$. The most used drugs to treat mothers were boosted protease inhibitors ( $99 \%$ cases).

\section{Conclusion}

In the studied patients we found high risk of prematurity in babies exposed in utero to antiretrovirals, but no association between prematurity and HIV infection in children or mother being part of Romanian cohort.

\section{Acknowledgement}

This work is supported by the Sectorial Operational Programme Human Resources Development financed from the European Social Found and by Romanian Government under contract number POSDRU/159/1.5/S/137390.

\section{Authors' details}

${ }^{1}$ Carol Davila University of Medicine and Pharmacy, Bucharest, Romania. ${ }^{2}$ National Institute for Infectious Diseases "Prof. Dr. Matei Balş", Bucharest, Romania. ${ }^{3}$ The Institute for Mother and Child Protection "Alfred Rusescu", Bucharest, Romania.

Published: 15 October 2014 